\title{
A NEW NOTION OF SEMICHARACTERS
}

\author{
BY \\ DENNISON R. BROWN AND MICHAEL FRIEDBERG( ${ }^{(1)}$
}

0. Introduction. A semigroup $S$ is (uniquely) divisible if, for each $x \in S$, and each positive integer $n$, there exists (a unique) $y \in S$ such that $y^{n}=x$. In the unique case we write $y=x^{1 / n}$. Uniquely divisible commutative semigroups, referred to in the sequel as UDC semigroups, have been characterized in [7]. Compact topological semigroups satisfying this hypothesis have been studied in [6], [11], [12], and [13]. Material of a related nature occurs in [15] and [17].

In [6], the authors showed that if $S$ is a finite-dimensional compact UDC semigroup in which the set of idempotents is totally disconnected, then there exist sufficiently many continuous homomorphisms (semicharacters) of $S$ into the complex unit disk to separate points. This usage of the complex disk as a range space is in line with the classical philosophy, "Abelian group is to the circle group as Abelian semigroup is to the complex unit disk semigroup." A large amount of work has been done on the investigation of this analogy; in particular, see [9] for a comprehensive survey of the algebraic results in this direction. For the case of Abelian topological semigroups and continuous semicharacters, see [16] and [23].

However, it is clear that the idempotent structure of the complex disk makes it unsuitable as a range space for continuous homomorphisms on general Abelian topological semigroups. In particular, if $S$ is a connected topological semilattice, then any continuous homomorphism of $S$ into the complex disk must be trivial. This deficiency is well known, and reasonable substitutes for the complex disk have been sought for some time.

If each maximal group of $S$ is trivial, then one of the most appealing replacements for the disk is some form of "thread"-a semigroup on a space homeomorphic to the unit interval in which one endpoint acts as an identity and the other as a zero. The complete structure of threads is given in [21], and questions concerning their suitability as range spaces for continuous semicharacters are raised therein. The basic building blocks for threads are $U$, the interval $[0,1]$ under real number multiplication; $M$, the interval $[0,1]$ under multiplication $x y=\min \{x, y\}$; and $C$, the interval $[1 / 2,1]$ under multiplication $x \cdot y=\max \{1 / 2, x y\}$, where $x y$ represents the ordinary real number product of $x$ and $y$.

The semigroup $U$ has been used successfully as a range space for a certain class of compact UDC semigroups in [6]. The semigroup $M$ is a very logical range space for the category of compact topological semilattices; the problem of whether every

Received by the editors June 14, 1968.

(1) Research by the first author supported in part by NASA Grant NGR-44-005-037. 
compact semilattice has enough continuous homomorphisms into $M$ to separate points remains unsolved. However, recent results of Lawson [20], based partially on earlier work of L. W. Anderson, [1] and [2], show that a large class of compact semilattices do have this property.

Unfortunately, threads are also deficient as range spaces, even for compact UDC semigroups having trivial maximal groups. As is pointed out in [3], the following semigroup on a two cell has no one-dimensional continuous homomorphic image: let $S$ be the Rees quotient of $U \times M$ modulo the ideal consisting of those pairs having at least one zero coordinate. Since $S$ contains an arc of idempotents, it is clear that the complex disk is also an unsuitable range space for $S$. Indeed, every nontrivial uniquely divisible continuous homomorphic image of $S$ can be shown to be iseomorphic to $S$. Consequently, any candidate for a uniquely divisible range space must contain a copy of $S$. However, since each maximal group of $S$ is trivial, it is clear that $S$ itself is not a satisfactory range space.

With the above in mind, let $Q$ be the universal solenoidal group [10], and let $B$ be the Rees quotient of the semigroup $U \times M \times Q$ modulo the ideal $J$ of all triples $(u, m, q)$ such that $u=0$ or $m=0$. The purpose of this paper is to demonstrate that the semigroup $B$ is a uniquely divisible range space for a large class of compact UDC semigroups.

On the other hand, the semigroup $B$ is not a satisfactory range space for the class of all compact Abelian semigroups. For example, the semigroup $C$ defined above has no nontrivial continuous homomorphisms into $B$. A more reasonable candidate for a range space for this class can be obtained by replacing $U$ by $C$ and $Q$ by the circle group in the definition of $B$. We reinforce this notion by showing that $B$ has sufficiently many continuous homomorphisms into this semigroup to separate points.

1. Preliminaries. We shall have occasion to use the following concept due to C. E. Clark [8].

Definition 1. Let $\left\{I_{1}, \ldots, I_{n}\right\}$ be a family of subsemigroups of a commutative semigroup $S$, each iseomorphic to $[0,1]$ under real number multiplication, and having a common identity and a common zero. The collection $\left\{I_{1}, \ldots, I_{n}\right\}$ is algebraically independent if there does not exist a partition of the integers $\{1, \ldots, n\}$ into disjoint, nonempty subsets $A$ and $B$ such that

$$
\prod_{j \in A} I_{j} \cap \prod_{j \in B} I_{j}
$$

contains a nonidempotent element.

It has been shown in [11] that, if each group in $S$ is trivial, and if $x \notin E$, then $[x]=\left\{x^{r}: r \text { is a positive rational number }\right\}^{*}$ is iseomorphic to $[0,1]$ under real number multiplication. We shall refer to any such semigroup as a usual unit interval. Hence, if $t$ is any nonnegative real number, then $x^{t}$ is a well defined element of $[x]$. 
It follows readily that the standard rules of exponents are satisfied, and we shall use these without further comment.

If $I$ is an ideal in a semigroup $S$, then the Rees quotient semigroup $S / I$ is the semigroup derived by shrinking $I$ to a single point which behaves as a zero; if $I$ is closed and $S$ is a compact topological semigroup, then it is well known that $S / I$ is a topological semigroup.

We adhere to the usual conventions for topological semigroups as set forth in [24]. Specifically, $A^{*}$ will denote the topological closure of the set $A$, a clan will be a compact, connected topological semigroup with identity, $H(e)$ will be the maximal subgroup containing an idempotent $e, H=\bigcup\{H(e): e \in E(S)\}$, where $E(S)$ is the set of idempotents of $S$. The empty set will be denoted by $\square$. The natural quasi order on $S$ is defined by $x \leqq y$ if and only if $x \in y S$. Indeed, $S$ is naturally totally ordered if the natural quasi order is antisymmetric and a total order. A semilattice is a commutative, idempotent semigroup. If $S$ is a semilattice, then the natural quasi order on $S$ is a partial order in which g.l.b. $\{x, y\}=x y$.

If $S$ is a compact semigroup, then $\mathscr{S}(S)$, the space of closed subsets of $S$ topologized in the usual manner, is known to be a compact topological semigroup under the induced multiplication of sets. Let $P^{*}$ denote the nonnegative real numbers and $N$ the negative real numbers. A subset $S$ of Euclidean $n$-space is a positive cone (or simply a cone) if $S$ is an additive subsemigroup of $E$ such that $P^{*} S \subseteq S$ and $N S \cap S$ $=\{0\}$.

It should be noted that $S$ is not assumed to be metric in any of the main theorems; dimension, when used in the topological sense, will mean inductive dimension. An iseomorphism is a homeomorphism and an isomorphism.

LEMMA 1. If $S$ is a compact UDC semigroup, and if $\{e\} \cup F \subseteq E(S)$, then $S e / S F e$ is a compact UDC semigroup.

Proof. The only property to be checked is the uniqueness of divisibility in $\mathrm{Se} / \mathrm{SFe}$. Let $\tau$ represent the natural map of $\mathrm{Se}$ onto $\mathrm{Se} / \mathrm{SFe}$, and suppose $\tau\left(x^{n}\right)$ $=\tau(y)^{n}$. If $x^{n}=y^{n}$, then it is clear that $\tau(x)=\tau(y)$. If $x^{n} \neq y^{n}$, then $\tau\left(x^{n}\right)=\tau\left(y^{n}\right)$ implies $x^{n}, y^{n} \in S F e$. If $x^{n}=s f e, f \in F$, then $x=s^{1 / n}=s^{1 / n}(f e)^{1 / n} f e \in S F e$. Similarly, $y \in S F e$, whence $\tau(x)=\tau(y)$.

If $X$ and $Y$ are topological semigroups, then by $\operatorname{Hom}(X, Y)$ we mean the set all continuous homomorphisms of $X$ into $Y$. The statement "Hom $(X, Y)$ separates points" means for each pair of distinct elements $a, b \in X$, there exists $f \in \operatorname{Hom}(X, Y)$ such that $f(a) \neq f(b)$.

LeMMA 2. Let $E$ be a compact topological semilattice, and let $F$ be a continuous homomorphic image of $E$. If $\operatorname{Hom}(E, M)$ separates points, then $\operatorname{Hom}(F, M)$ separates points.

Proof. This is proved in [20]. 
If $S$ is a compact uniquely divisible semigroup, then each of the sequences $\left\{x^{1 / n}\right\}$ and $\left\{x^{n}\right\}$ contain unique idempotent cluster points ([11] and [18]). Denote the idempotent cluster point of $\left\{x^{1 / n}\right\}$ by $1(x)$ and that of $\left\{x^{n}\right\}$ by $0(x)$. Note that, although $1(x)$ is an identity for $x, 0(x)$ need not be a zero for $x$.

LEMMA 3. Let $x, y, z \in S$, a compact UDC semigroup. Then

(a) $1(x) 1(y)=1(x y)$;

(b) $0(x) 0(y)=0(x y)$;

(c) if $x y=x z$, then $1(x) y=1(x) z$ and $0(x) y=0(x) z$;

(d) if $x y=x z$ and $y, z \in S 1(x)$, then $y=z$.

Proof. Parts (a) and (b) follow from continuity of multiplication in $S$ and the identities $x^{1 / n} y^{1 / n}=(x y)^{1 / n}$ and $x^{n} y^{n}=(x y)^{n}$. For part (c), note $x y^{2}=(x y) y=(x z) y$ $=(x y) z=x z^{2}$, whence $x^{1 / 2} y=x^{1 / 2} z$. Inductively, $x^{1 / n} y=x^{1 / n} z$, and continuity of multiplication yields $1(x) y=1(x) z$. The other equality follows similarly from the equations $x^{n} y=x^{n} z$. Part (d) is an immediate consequence of the first portion of part (c).

Two elements $a$ and $b$ of a semigroup $S$ are $\mathscr{H}$-related if they generate the same principal left. and right ideals. It is easily seen that this is an equivalence relation on $S$, and if $S$ is commutative, then it is a congruence. If, in addition, $S$ is a compact topological semigroup, then it is well known that the quotient space $S / \mathscr{H}$ is a topological semigroup in which $H=E(S / \mathscr{H})$. This relation was first presented by J. A. Green; for details of its algebraic properties see [9]. The topological version has been studied extensively in [4] and [5]. We reserve the letter $\phi$ for the natural map from $S$ to $S / \mathscr{H}$. It is easily seen that if $S$ is compact and Abelian, and if $a$ and $b$ are $\mathscr{H}$-related, then there exists $h \in H$ such that $a=b h$.

Lemma 4. Let $S$ be a compact Abelian semigroup, and let $T=S / \mathscr{H}$, and let $F$ be a closed subset of $E(T)$. Then the Rees quotient semigroup $T / T F$ is iseomorphic to the $\mathscr{H}$-class quotient semigroup of $S / S\left(\phi^{-1}(F) \cap E(S)\right)$.

Proof. Consider the diagram

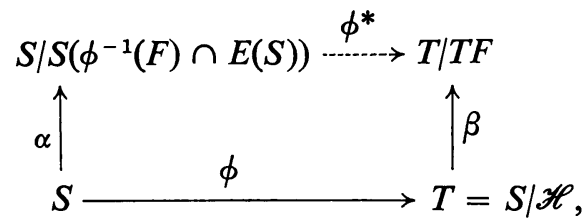

where $\phi^{*}$ is induced by $\phi$ and the natural maps $\alpha$ and $\beta$. It is easily established by diagram chasing that if $x$ and $y$ are two elements of $S / S\left(\phi^{-1}(F) \cap E(S)\right)$, then $\phi^{*}(x)=\phi^{*}(y)$ if and only if $x$ and $y$ are $\mathscr{H}$-related.

2. Proof of the main theorem. We construct a continuous homomorphism of $S$ into $B$ separating two arbitrary distinct elements $x$ and $y$ of $S$ by simplifying $S$ in stages, relying primarily on Lemma 1 . It will often be necessary to work in $S / \mathscr{H}$, 
which has all the properties desired of $S$ save (perhaps) that of being finite dimensional. This possible anomaly of $S / \mathscr{H}$ in no way affects the argument, however.

THEOREM. Let $S$ be a compact finite-dimensional UDC topological semigroup in which Hom $(E(S), M)$ separates points. Then $\operatorname{Hom}(S, B)$ separates points $\left({ }^{2}\right)$.

Proof. The semigroup $S \times U / S \times\{0\}$ has all the properties mentioned in the hypotheses above and includes, as $S \times\{1\}$, an iseomorphic copy of $S$. Hence we may assume without loss of generality that $S$ is also connected and has a zero.

Now fix distinct elements $x$ and $y$ of $S$.

First reduction: We may assume $1(x)=1(y)$, and that $1(x)$ is an identity for $S$. Indeed, let $1(x)=e, 1(y)=f$, and assume $e \neq f$. Then $e f \neq e$ or $e f \neq f$. Assume $e f \neq e$, and note $1(x e)=1(x)=e$ and $1(y e)=e f$. By Lemma 1 , the semigroup $S e / S e f$ is a continuous homomorphic image of $S$ of the proper type, and the images of $x$ and $y$ in this semigroup remain separated. Further, since $y$ is mapped into the zero of this semigroup, it follows that any homomorphism of $\mathrm{Se} / \mathrm{Sef}$ into $B$ separating the image of $x$ and zero will, by composition with the natural map from $S$ to $S e / S e f$, separate $x$ and $y$. Hence, if $x \neq e$, then it is sufficient to separate the images of $x$ and $x^{2}$. If, on the other hand, $x=e$, then any nontrivial homomorphism of $\mathrm{Se} / \mathrm{Sef}$ into $B$ will separate the images of $x$ and $y$. If $\mathrm{Se} / \mathrm{Sef}$ consists only of idempotents, then, by Lemma 2, Hom ( $\mathrm{Se} / \mathrm{Sef}, M)$ separates points and hence Hom $(\mathrm{Se} / \mathrm{Sef}, B)$ separates points. If there exists $z \in S e / S e f$ such that $z \neq z^{2}$, then it suffices to separate $z$ and $z^{2}$ in $B$.

Hence we may assume that $1(x)=1(y)=e$, and by passing from $S$ to $S e$ by translation we may assume that $e$ is the identity of $S$.

Second reduction: We may assume that, if $f \in E(S) \mid\{0\}$, then $x f \neq y f$. In particular, set $F=\{f \in E(S): x f=y f\}$. Then, by Lemma 1, the semigroup $S / S F$ has the properties required in the hypotheses of the theorem. Clearly neither $x$ nor $y$ belongs to $S F$, and thus the images of these elements in $S / S F$ are separated. Finally, the algebraic properties of the Rees quotient semigroup show that multiplication by a nonzero idempotent in $S / S F$ must leave the images of $x$ and $y$ separated. Hence, by renaming $S / S F$ as $S$, we accomplish the second reduction.

Third reduction: For each nonzero idempotent e, we may assume $H(e)$ is iseomorphic to $H(1)$ under the translation homomorphism $x \rightarrow x e$. To this end, choose $e \in E(S), e \neq 0$, such that $H(e)$ has maximum dimension among the groups of $S$. By way of the translation homomorphism, we may assume that $e=1$ and hence that $H(1)$ has maximum dimension among the groups of $S$. It is well known that any compact Abelian uniquely divisible topological group is iseomorphic to the Cartesian product of some number of copies of $Q$, the universal solenoidal group [10]. Since $H(1)$ is finite dimensional, it follows that only finitely many copies of $Q$ are needed to form $H(1)$. Since $Q$, as the inverse limit of circle groups, has no

$\left({ }^{2}\right)$ We are indebted to the referee for an instructive example showing that one step in the original proof required amplification. 
proper nontrivial connected subgroups, it follows that there exists a relative neighborhood $N$ of 1 in $H(1)$ such that $N$ contains no nontrivial connected subgroup of $H(1)$.

Now, for each $e \in E(S)$, let $C(e)=\{g \in H(1): g e=e\}$. Note that $C(1)=\{1\}$, $C(0)=H(1)$, and each $C(e)$ is a compact divisible (hence connected) subgroup of $H(1)$. Let $F=\{e \in E(S): C(e) \neq\{1\}\}$. To see that $F$ is a closed subset of $S$, let $\left\{e_{\alpha}\right\}$ be a net in $F$ converging to $e$. For each $\alpha$, choose $g_{\alpha} \in C\left(e_{\alpha}\right) \backslash N$; the net $\left\{g_{\alpha}\right\}$ must cluster to some element $g$ in $H(1) \backslash N$. By continuity of multiplication, $g e=e$, whence $e \in F$.

Finally, if $e \in E(S) \backslash F$, then $C(e)=\{1\}$; since $C(e)$ is the kernel of the continuous group homomorphism defined by translation by $e$, it follows directly that this mapping is an iseomorphism of $H(1)$ into $H(e)$. Since $H(1)$ has maximal dimension among the groups of $S$, the quotient group $H(e) / e H(1)$ is a connected zero-dimensional group and hence is trivial. It is immediate that the iseomorphism mentioned above maps $H(1)$ onto $H(e)$.

To complete the third reduction, form the semigroup $S / S F$. The nonzero maximal groups of this semigroup are iseomorphic copies of the maximal groups in $S$ which are associated with idempotents in $E(S) \backslash F$, and the algebra of the Rees quotient semigroup assures that $S / S F$ inherits the properties set forth in hypotheses together with the ones accomplished by the first two reductions. Since $x$ and $y$ are not elements of $S F$, it is clear that their images remain separated in $S / S F$.

Fourth reduction: We may assume, if $x \in S \backslash H$, that $0(x)=0$. Note that the semigroup $S / \mathscr{H}=T$ satisfies all the hypotheses of the theorem except possibly that of being finite dimensional, and that the natural homomorphism $\phi: S \rightarrow T$ is a closed monotone map. In the ensuing paragraph, we work exclusively in $T$.

Specifically, we show there exists an idempotent $e \in T$ and a closed subset $C$ of idempotents of $T e \backslash\{e\}$ such that if $z \in T e \mid E(T)$, then $0(z) \in C$. If there exists a nonzero idempotent $e \in T$ such that $T e$ is all idempotents, then this condition is vacuously satisfied for the closed set $C=\{0\}$. Otherwise, choose $z \in T \backslash E(T)$. If the pair $(1(z), 0(z) \cdot E(T))$ fails to satisfy the desired properties of $e$ and $C$, then there exists $w \in T \cdot 1(z) \backslash E(T)$ such that $0(w) \notin 0(z) \cdot E(T)$. The elements $w$ and $w z$ are distinct nonidempotents, since $0(w z)=0(w) 0(z)<0(w)$ and $1(w z)=1(w) 1(z)=1(w)$. Proceeding inductively, we assume the existence of $k$ distinct nonidempotents $z_{1}, z_{2}, \ldots, z_{k}$ such that $1\left(z_{1}\right)=1\left(z_{2}\right)=\cdots=1\left(z_{k}\right)=f$ and $0\left(z_{1}\right)<0\left(z_{2}\right)<\cdots<0\left(z_{k}\right)$. For $i, j$ distinct integers between 1 and $k$, put $A(i, j)=\left\{g \in f E(T): g 0\left(z_{i}\right)=g 0\left(z_{j}\right)\right\}$. Since each $A(i, j)$ is a closed set, $A=\bigcup\{A(i, j): i \neq j ; i, j=1,2, \ldots, k\}$ is a closed subset of $f E(T)$ and $f \notin A$. If the pair $(f, A)$ fails to satisfy the properties desired, then there exists $z_{k+1} \in f T \mid E(T)$ such that $0\left(z_{k+1}\right) \notin A$. The nonidempotent elements $w_{1}=z_{1} z_{k+1}, w_{2}=z_{2} z_{k+1}, \ldots, w_{k}=z_{k} z_{k+1}, w_{k+1}=z_{k+1}$ then satisfy $1\left(w_{1}\right)$ $=1\left(w_{2}\right)=\cdots=1\left(w_{k+1}\right)$ and $0\left(w_{1}\right)<0\left(w_{2}\right)<\cdots<0\left(w_{k+1}\right)$.

Now suppose $\operatorname{dim} S=n$. Under the assumption that there does not exist a pair $(e, C)$ of the desired type, we obtain by the above method a family $z_{1}, z_{2}, \ldots, z_{n+1}$ 
of nonidempotents in $T$ satisfying $1\left(z_{1}\right)=1\left(z_{2}\right)=\cdots=1\left(z_{n+1}\right)$ and $0\left(z_{1}\right)<0\left(z_{2}\right)$ $<\cdots<0\left(z_{n+1}\right)$. For each integer $i$, the semigroup $\left[z_{i}\right]$ in $T$ is iseomorphic to $U$, and the semigroup $\phi^{-1}\left(\left[z_{i}\right]\right)$ is therefore a subclan of $S$ having only two idempotents. Let $h$ be the idempotent such that $\phi(h)=1\left(z_{i}\right)$. In each $\phi^{-1}\left(\left[z_{i}\right]\right)$ we may select a local one parameter semigroup $\sigma_{i}$ meeting $H(h)$ only in $h$, [22]. Let $W$ be the Cartesian product of $n+1$ copies of $[0,1]$, and define $F: W \rightarrow S$ by

$$
F\left(r_{1}, r_{2}, \ldots, r_{n+1}\right)=\sigma_{1}\left(r_{1}\right) \sigma_{2}\left(r_{2}\right) \cdots \sigma_{n+1}\left(r_{n+1}\right) .
$$

The function $F$ is clearly continuous; we show that it is also one-to-one. Suppose $\sigma_{1}\left(r_{1}\right) \sigma_{2}\left(r_{2}\right) \cdots \sigma_{n+1}\left(r_{n+1}\right)=\sigma_{1}\left(s_{1}\right) \sigma_{2}\left(s_{2}\right) \cdots \sigma_{n+1}\left(s_{n+1}\right)$. Reorder the family $\left\{\sigma_{i}\right\}$ so that $r_{i} \leqq s_{i}, i=1,2, \ldots, k$ and $r_{i}>s_{i}, i=k+1, k+2, \ldots, n+1$, and assume $k<n+1$. Let $t_{i}=s_{i}-r_{i}, i=1,2, \ldots, k$, and $p_{i}=r_{i}-s_{i}, i=k+1, k+2, \ldots, n+1$. We then have

$$
\sigma_{1}\left(r_{1}\right) \sigma_{2}\left(r_{2}\right) \cdots \sigma_{k}\left(r_{k}\right) \sigma_{k+1}\left(s_{k+1}\right) \cdots \sigma_{n+1}\left(s_{n+1}\right) \sigma_{k+1}\left(p_{k+1}\right) \cdots \sigma_{n+1}\left(p_{n+1}\right)
$$

equal to

$$
\sigma_{1}\left(r_{1}\right) \sigma_{2}\left(r_{2}\right) \cdots \sigma_{k}\left(r_{k}\right) \sigma_{k+1}\left(s_{k+1}\right) \cdots \sigma_{n+1}\left(s_{n+1}\right) \sigma_{1}\left(t_{1}\right) \cdots \sigma_{k}\left(t_{k}\right) .
$$

Set $a$ equal to

$$
\sigma_{1}\left(r_{1}\right) \sigma_{2}\left(r_{2}\right) \cdots \sigma_{k}\left(r_{k}\right) \sigma_{k+1}\left(s_{k+1}\right) \cdots \sigma_{n+1}\left(s_{n+1}\right) ;
$$

clearly $\left\{a^{1 / n}\right\}$ converges to $h$, so that by Lemma $3, \sigma_{k+1}\left(p_{k+1}\right) \sigma_{k+2}\left(p_{k+2}\right) \cdots$ $\sigma_{n+1}\left(p_{n+1}\right)=\sigma_{1}\left(t_{1}\right) \sigma_{2}\left(t_{2}\right) \cdots \sigma_{k}\left(t_{k}\right)$. By passing to the semigroup $T$ and taking powers, we conclude $0\left(z_{k+1}\right) 0\left(z_{k+2}\right) \cdots 0\left(z_{n+1}\right)=0\left(z_{1}\right) 0\left(z_{2}\right) \cdots 0\left(z_{k}\right)$, which is contradictory to the way in which $z_{1}, z_{2}, \ldots, z_{n+1}$ were chosen. Thus we must have $k=n+1$, whence

$$
\sigma_{1}\left(r_{1}\right) \sigma_{2}\left(r_{2}\right) \cdots \sigma_{n+1}\left(r_{n+1}\right)=\sigma_{1}\left(r_{1}\right) \sigma_{2}\left(r_{2}\right) \cdots \sigma_{n+1}\left(r_{n+1}\right) \sigma_{1}\left(t_{1}\right) \sigma_{2}\left(t_{2}\right) \cdots \sigma_{n+1}\left(t_{n+1}\right) .
$$

Again we resort to Lemma 3 to conclude $\sigma_{1}\left(t_{1}\right) \sigma_{2}\left(t_{2}\right) \cdots \sigma_{n+1}\left(t_{n+1}\right)=h$, whence each $t_{2}=0$. This shows that $F$ is one-to-one.

Since $F$ is a homeomorphism, the image of $W$ is an $n+1$ cell in $S$, which contradicts our assumption that $S$ is $n$ dimensional. Hence there must exist a pair $(e, C)$ in $T$ such that $z \in T e \backslash E(T)$ implies $0(z) \in C$.

To accomplish the fourth reduction, we now set $f=\phi^{-1}(e) \cap E(S), D=\phi^{-1}(C)$ $\cap E(S)$. It is now immediate that the semigroup $S f / S D$ has the desired property, as well as the properties achieved in reductions one, two, and three.

For each $e \in E(T)$, set $P(e)=\{z \in T: 1(z)=e\} \cup\{0\}$. Note that $P(e)$ is a connected divisible subsemigroup of $T$ with identity $e$, zero, and no other idempotents.

For each $e \in E(T), P(e)$ is a closed set. If $z \in P(e) \backslash E(T)$, then $[z]$ is iseomorphic to $U$; it follows from the arguments in the fourth reduction that, if $\operatorname{dim} S=n$, then in $P(e)$ there are at most $n$ algebraically independent semigroups of the form $[z]$. Select a maximal family $\left[z_{1}\right],\left[z_{2}\right], \ldots,\left[z_{k}\right]$ with this property. The semigroup $R=\left[z_{1}\right]\left[z_{2}\right] \cdots\left[z_{k}\right]$ has been characterized in [6] as a $k$-cell whose bounding $k-1$ 
sphere $V$ consists of $e, 0$, and all elements $z_{1}^{r_{1}} z_{2}^{r_{2}} \cdots z_{k}^{r_{k}}$ such that at least one $r_{i}=0$. Furthermore, the element $z_{0}=z_{1} z_{2} \cdots z_{k} \in R \mid V$, and $\left[z_{0}\right] \cap V=\{e, 0\}$. It follows directly from arguments involving algebraic independence as exemplified in the proof of Theorem 1.5 of [6] that, for each $p \in P(e) \backslash V$, there exists $q \in P(e) \backslash E(T)$ such that $q \in[p] \cdot\left[z_{0}\right] \cap V$. Now let $\left\{p_{\alpha}\right\}$ be a net in $P(e)$ converging to $p_{0}$. Since $R$ is closed, we need only consider the case in which $\left\{p_{\alpha}\right\}$ is eventually in $P(e) \backslash R$. Hence, for sufficiently large $\alpha$, there exist $q_{\alpha} \in P(e) \backslash E(T)$ such that $q_{\alpha} \in\left[p_{\alpha}\right] \cdot\left[z_{0}\right] \cap V$, and thus $\left[q_{\alpha}\right] \subset\left[p_{\alpha}\right] \cdot\left[z_{0}\right] \cap V$.

By appropriate choice of subnets, we may assume the nets $\left\{\left[p_{\alpha}\right]\right\}$ and $\left\{\left[q_{\alpha}\right]\right\}$ converge in $\mathscr{S}(S)$ to $J$ and $L$ respectively. It is well known [19] that each of $J$ and $L$ is an arc semigroup having $e$ and 0 as endpoints. Since $L \subset V, L$ is iseomorphic to $U$. By continuity of multiplication in $\mathscr{S}(S), L \subseteq J \cdot\left[z_{0}\right] \cap V$. Let $k \in L \backslash E(T)$, $k=h z, h \in J, z \in\left[z_{0}\right]$. By Lemma 3, $1(k)=1(h) 1(z)$, whence $1(h)=e$. If $h=e$, then $k=z \in(V \backslash E(T)) \cap\left[z_{0}\right]$, which is false. Hence $h \neq e$, and $[h]$ is a nondegenerate subsemigroup of $J$. Since $\left\{h^{n}\right\}$ converges to 0 , and $\left\{h^{1 / n}\right\}$ converges to $e$, it follows directly that $[h]=J$. Since $p_{0} \in J$, it is now clear that $p_{0} \in P(e)$, which completes the proof of the claim.

Hence $P(e)$ is iseomorphic to the one point compactification of a $k$-dimensional closed cone in $R^{n}$ [6, Theorem 1.5]. Choose $e \in E(T)$ such that $P(e)$ has maximal dimension. By use of the homomorphism induced by translation by $e$, we may assume that $e=1$. Let, as before, $\left[z_{1}\right],\left[z_{2}\right], \ldots,\left[z_{k}\right]$ be a maximal family of algebraically independent subsemigroups of $P(1)$, and set $R=\left[z_{1}\right] \cdot\left[z_{2}\right] \cdots\left[z_{k}\right]$.

Fifth reduction: We may assume, for each $e \in E(T) \mid\{0\}$, that $R$ is iseomorphic to $R e$ under the restriction of the mapping induced by multiplication by $e$. Set $F=\{e \in$ $E(T)$ : there exist $a, b \in R, a \neq b$ such that $e a=e b\}$. It is easy to see that $F$, and hence $F^{*}$, is an ideal in $E(T)$. We show $1 \notin F^{*}$; for suppose $\left\{e_{\alpha}\right\}$ is a net in $F$ converging to 1 . Let $\left\{a_{\alpha}\right\}$ and $\left\{b_{\alpha}\right\}$ be nets in $R$ such that $e_{\alpha} a_{\alpha}=e_{\alpha} b_{\alpha}$. For each $\alpha$, let $a_{\alpha}=$ $z_{1}^{r(\alpha, 1)} z_{2}^{r(\alpha, 2)} \cdots z_{k}^{r(\alpha, k)}$ and let $b_{\alpha}=z_{1}^{s(\alpha, 1)} z_{2}^{s(\alpha, 2)} \cdots z_{k}^{s(\alpha, k)}$. By using Lemma 3 as in the fourth reduction, we may "cancel" each $z_{i}$ from one side or the other of the equations $e_{\alpha} a_{\alpha}=e_{\alpha} b_{\alpha}$ to obtain nonnegative real numbers $p(i, \alpha)$ and decompositions of the integers $1,2, \ldots, k$ into disjoint sets $M(\alpha)$ and $N(\alpha)$ such that

$$
e_{\alpha} \cdot \prod_{i \in M(\alpha)} z_{i}^{p(i, \alpha)}=e_{\alpha} \cdot \prod_{i \in N(\alpha)} z_{i}^{p(i, \alpha)}
$$

Note that, since $a_{\alpha} \neq b_{\alpha}$ for each $\alpha$, we have at least one $p(i, \alpha)>0$. Let $p(\alpha)$ $=\sum_{i=1}^{k} p(i, \alpha)$, and set $q(i, \alpha)=p(i, \alpha) / p(\alpha)$. Finally, choose a subnet (of the same name) such that $M(\alpha)=M(\beta)=M$ and $N(\alpha)=N(\beta)=N$ for all $\alpha$ and $\beta$, and let $\{q(i, \alpha)\}$ converge to $q(i)$ for each $i=1,2, \ldots, k$. By continuity of multiplication we then have

$$
1 \cdot \prod_{i \in M} z_{i}^{q(i)}=1 \cdot \prod_{i \in N} z_{i}^{q(i)}
$$


But $\sum_{i=1}^{k} q(i)=1$, which contradicts the algebraic independence of the set of subsemigroups $\left\{\left[z_{i}\right]\right\}$. Hence $1 \notin F^{*}$.

The quotient semigroup $T / T F^{*}$ is clearly a semigroup of the type desired in the fifth reduction. Furthermore, $T / T F^{*}$ is iseomorphic to the $\mathscr{H}$-class quotient semigroup of $S / S\left(\phi^{-1}\left(F^{*}\right) \cap E(S)\right)$ by Lemma 4 . This procedure accomplishes the fifth reduction.

We continue to let $R=\left[z_{1}\right] \cdot\left[z_{2}\right] \cdots\left[z_{k}\right]$, where $\left[z_{1}\right],\left[z_{2}\right], \ldots,\left[z_{k}\right]$ is a maximal family of algebraically independent subsemigroups of $P(1)$. By an argument similar to one given in reduction four, we may choose, for each integer $i=1,2, \ldots, k$, $x_{i} \in \phi^{-1}\left(\left[z_{i}\right]\right)$ such that $\left[x_{i}\right]$ is iseomorphic to $U$. Let $Y=\left[x_{1}\right] \cdot\left[x_{2}\right] \cdots\left[x_{k}\right]$, and note $\phi$ maps $Y$ iseomorphically onto $R$.

If $s \in S \backslash E(S)$, then there exist $a, b \in Y \backslash\{0\}, g \in H(1)$ such that $s a=b 1(s) g$. If $\phi(s) \in E(T)$, then $s \in H(1(s))$, so that by reduction three there exists $g \in H(1)$ with $s=1(s) g$. If $\phi(s) \in T \backslash E(T)$, then let $e=1(\phi(s))$. From the fifth reduction, $\left[z_{1} e\right]$, $\left[z_{2} e\right], \ldots,\left[z_{k} e\right]$ form an algebraically independent family in $P(e)$; this family is maximal since $P(1)$ has been chosen to have maximum dimension. Hence the set $\{1,2, \ldots, k\}$ may be decomposed into disjoint subsets $M$ and $N$ such that

$$
\phi(s) \cdot \prod_{i \in M} z_{i}^{r(i)} \cdot e=\prod_{i \in N} z_{i}^{r(i)} \cdot e .
$$

It follows that, in $S$, the elements $s \cdot \prod_{i \in M} x_{i}^{r(i)}$ and $\prod_{i \in N} x_{i}^{r(i)} \cdot 1(s)$ are $\mathscr{H}$-related. Hence, for some $h \in H(1(s))$,

$$
s \cdot \prod_{i \in M} x_{i}^{r(i)}=\prod_{i \in N} x_{i}^{r(i)} \cdot 1(s) h .
$$

However, from the third reduction; $h=1(s) g$ for some $g \in H(1)$, and the claim is proved.

If $s a=b 1(s) g$ and $s c=d 1(s) h$, where $a, b, c, d \in Y$ and $g, h \in H(1)$, then $a d=b c$ and $g=h$. Indeed, $s a c=b c 1(s) g=a d 1(s) h$, whence $\phi(b c) \phi(1(s))=\phi(a d) \phi(1(s))$. By the fifth reduction, $\phi(b c)=\phi(a d)$, and since $\phi$ is an iseomorphism on $Y$, it follows that $b c=a d$. The semigroup $Y H(1(s))$ is a compact divisible subsemigroup of $S$ with 0 and $1(s)$ as its only idempotents, hence has nonzero cancellation by Lemma 3. This fact yields $1(s) g=1(s) h$, and by the third reduction, $g=h$.

We are now prepared to exhibit a one-to-one continuous function of $S \mid\{0\}$ into $R^{k} \times E(S) \times H(1)$. Let $\mu$ be an iseomorphism of $P(1) \backslash\{0\}$ into $R^{k}$ [6, Theorem 1.5]. Define $\psi: S \backslash\{0\} \rightarrow R^{k} \times E(S) \times H(1)$ by

$$
\begin{aligned}
\psi(s) & =(0, s, 1) & & \text { if } s \in E(S), \\
& =(\mu \phi(b)-\mu \phi(a), 1(s), g) & & \text { if } s \in S \backslash E(S), s a=b 1(s) g .
\end{aligned}
$$

It is clear that $\psi$ is well defined on $E(S)$. If $s a=b 1(s) g$ and $s c=d 1(s) g$, then, since $a d=b c$, we have

$$
\mu \phi(a)+\mu \phi(d)=\mu \phi(b)+\mu \phi(c),
$$

from which it follows that $\psi$ is well defined on $S \backslash E(S)$. 
The function $\psi$ restricted to each semigroup of the form $\phi^{-1}(P(e)) \mid\{0\}, e \in E(T)$, is precisely the function $\gamma$ defined in Theorem 2.2 of [6]. Hence it is a continuous isomorphism of $\phi^{-1}(P(e)) \mid\{0\}$ into $R^{k} \times\{e\} \times H(1)$. It now follows that $\psi$ is one-toone, since $\psi(x)=\psi(y)$ requires $1(x)=1(y)$, so that $x$ and $y$ both belong to $\phi^{-1}(P(\phi(1(x)))$, whence $x=y$.

It is immediate that $\psi$ is continuous on $E(S) \mid\{0\}$. Let $\left\{s_{\alpha}\right\}, \alpha \in D$, be a net in $S \backslash E(S)$ converging to $s_{0} \in S \backslash\{0\}$. Set $s_{\alpha} a_{\alpha}=b_{\alpha} 1\left(s_{\alpha}\right) g_{\alpha}$, where $a_{\alpha}, b_{\alpha} \in Y \backslash\{0\}$, and $g_{\alpha} \in H(1)$. By judicious choice of subnets we may assume the nets $\left\{a_{\alpha}\right\},\left\{b_{\alpha}\right\}$, $\left\{1\left(s_{\alpha}\right)\right\}$, and $\left\{g_{\alpha}\right\}$ converge respectively to $a, b, e$, and $g$, where $a, b \in Y, e \in E(S)$, and $g \in H(1)$. Note that $e \neq 0$, since otherwise $\left\{s_{\alpha}\right\}=\left\{s_{\alpha} \cdot 1\left(s_{\alpha}\right)\right\}$ converges to 0 , which is false. Also, $e 1\left(s_{0}\right)=1\left(s_{0}\right)$, since, for each $n$, the equality $e s_{0}^{1 / n}=s_{0}^{1 / n}$ follows from the convergence of the net $\left\{1\left(s_{\alpha}\right) s_{\alpha}^{1 / n}\right\}=\left\{s_{\alpha}^{1 / n}\right\}$.

We show next that $a$ and $b$ are not zero. For each $\alpha \in D$, set

$$
a_{\alpha}=\prod_{(i, \alpha) \in M(\alpha)} x_{i}^{r(i, \alpha)}, \quad b_{\alpha}=\prod_{(i, \alpha) \in N(\alpha)} x_{i}^{r(i, \alpha)},
$$

where $\{M(\alpha), N(\alpha)\}$ is a decomposition of the integers $1,2, \ldots, k$. By again resorting to subnets, we assume $M(\alpha)=M(\beta)=M$ and $N(\alpha)=N(\beta)=N$ for $\alpha, \beta \in D$. We then have in $T=S / \mathscr{H}$ the equations

$$
\phi\left(s_{\alpha}\right) \cdot \prod_{(i, \alpha) \in M} z_{i}^{r(i, \alpha)}=\prod_{(i, \alpha) \in N} z_{i}^{r(i, \alpha)} \cdot \phi\left(1\left(s_{\alpha}\right)\right) .
$$

Set $r(\alpha)=\sum_{i=1}^{k} r(i, \alpha), t(\alpha)=r(\alpha)^{-1}, v(i, \alpha)=r(i, \alpha) t(\alpha)$. Note $\sum_{i=1}^{k} v(i, \alpha)=1$ for each $\alpha \in D$. We now have

$$
\phi\left(s_{\alpha}\right)^{t(\alpha)} \cdot \prod_{(i, \alpha) \in M} z_{i}^{v(i, \alpha)}=\prod_{(i, \alpha) \in N} z_{i}^{v(i, \alpha)} \cdot \phi\left(1\left(s_{\alpha}\right)\right) .
$$

If the net $\{t(\alpha)\}$ clusters to zero in the real numbers, then let $\{t(\gamma)\}$ be a subnet converging to zero such that, for each $i,\{v(i, \gamma)\}$ converges to $v(i)$ and $\left\{\phi\left(s_{\gamma}\right)^{t(\gamma)}\right\}$ converges to $y_{0} \in T$. For each integer $n$, the elements $\phi\left(s_{\gamma}\right)^{1 / n}$ are eventually less than or equal to $\phi\left(s_{\gamma}\right)^{t(\gamma)}$ in the natural quasi order of $T$. Since the natural quasi order is a closed relation on $T$, it follows that for each integer $n, \phi\left(s_{0}\right)^{1 / n} \in T y_{0}$, so that $\phi\left(1\left(s_{0}\right)\right)=1\left(\phi\left(s_{0}\right)\right) \in T y_{0}$. Note that $y_{0} \in E(T)$, since otherwise $1\left(\phi\left(s_{0}\right)\right)=0$; hence $1\left(\phi\left(s_{0}\right)\right) y_{0}=1\left(\phi\left(s_{0}\right)\right)$.

From continuity of multiplication and equation $\left(^{*}\right)$, we now have

$$
y_{0} \cdot \prod_{i \in M} z_{i}^{v(i)}=\prod_{i \in N} z_{i}^{v(i)} \cdot \phi(e) .
$$

Upon multiplying both sides of the above equation by $1\left(\phi\left(s_{0}\right)\right)$, we get

$$
\phi\left(1\left(s_{0}\right)\right) \cdot \prod_{i \in M} z_{i}^{v(i)}=\prod_{i \in N} z_{i}^{v(i)} \cdot \phi\left(1\left(s_{0}\right)\right) .
$$

However, by reduction five, multiplication by $\phi\left(1\left(s_{0}\right)\right)$ is an iseomorphism on $R$. Since $\sum_{i=1}^{k} v(i)=1$, this contradicts the algebraic independence of the family 
$\left\{\left[z_{i}\right]\right\}$. Notice that this argument is valid even in the event that one of $M$ and $N$ is void.

Hence the net $\{t(\alpha)\}$ does not cluster to zero, and therefore the net $\{r(\alpha)\}$ is a bounded set of real numbers. It now follows that each net $\{r(i, \alpha)\}, \alpha \in D$, is bounded, and hence that the nets $\left\{a_{\alpha}\right\}$ and $\left\{b_{\alpha}\right\}$ can have only nonzero cluster points. Hence $a$ and $b$ are nonzero elements of $Y$.

We now have $s_{0} a=b e g$, where $a, b \in Y \backslash\{0\}, e \in E(S) \mid\{0\}$, and $g \in H(1)$. If $s_{0} \in S \backslash E(S)$, then convergence of $\left\{\psi\left(s_{\alpha}\right)\right\}$ to $\psi\left(s_{0}\right)$ depends only upon establishing $e=1\left(s_{0}\right)$. For each integer $n$ we have $\phi\left(s_{0}\right)^{1 / n} \phi(a)^{1 / n}=\phi(b)^{1 / n} \phi(e)$. Hence $\phi(e)$ $=1\left(\phi\left(s_{0}\right)\right)=\phi\left(1\left(s_{0}\right)\right)$; since $\phi$ is one-to-one on $E(S)$, the desired result is obtained.

If $s_{0} \in E(S)$, then it must be shown that $\left\{\mu \phi\left(b_{\alpha}\right)-\mu \phi\left(a_{\alpha}\right)\right\}$ converges to zero and that $g=1$. We establish the former by showing $a=b$. From $s_{0} a=b e g$ we get $\left(s_{0} e\right) a=\left(s_{0} e\right) b g$. Furthermore, $s_{0} e \neq 0$, since otherwise $\left\{s_{0} s_{\alpha}\right\}$ converges to zero, which is false. Hence $\phi\left(s_{0} e\right) \phi(a)=\phi\left(s_{0} e\right) \phi(b)$, which, by reduction five, implies $\phi(a)=\phi(b)$, and thus $a=b$.

To get $g=1$, note $s_{0} e a=\left(s_{0} e a\right) g=\left(s_{0} e a\right) g^{n}$, whence $s_{0} e a^{1 / n}=s_{0} e a^{1 / n} g$, and so $s_{0} e=s_{0} e g$. But, by reduction three, multiplication by a nonzero idempotent is an iseomorphism on $H(1)$, and so $g=1$. The continuity of $\psi$ has now been established in all cases.

The function $\psi$ is a homomorphism in the following sense: if $s, t$, and $s t$ are all nonzero elements of $S$, then $\psi(s) \psi(t)=\psi(s t)$. This is easily verified from the definition of $\psi$. However, even if $S \backslash\{0\}$ is a subsemigroup of $S$, it may be impossible to compactify the range of $\psi$ in such a way as to define a continuous multiplicative extension of $\psi$ on all of $S$. The remainder of the argument is concerned with a final alteration of $S$ to remove this problem.

Let $\pi$ be the projection of $R^{k} \times E(S) \times H(1)$ into $R^{k}$, and let $\left\{x_{\alpha}\right\}$ be a net in $S \backslash\{0\}$ converging to 0 . We show that if the net $\left\{1\left(x_{\alpha}\right)\right\}$ does not cluster to 0 in $S$, then the net $\left\{\pi\left(\psi\left(x_{\alpha}\right)\right)\right\}$ in $R^{k}$ must converge to $\infty$. Indeed, by choosing subnets as in reduction five, we may assume there is a decomposition $\{M, N\}$ of the integers $1,2, \ldots, k$, and there are nonnegative real numbers $p(i, \alpha)$ so that

$$
x_{\alpha} \prod_{i \in M} x_{i}^{p(i, \alpha)}=\prod_{i \in N} x_{i}^{p(i, \alpha)} 1\left(x_{\alpha}\right) g_{\alpha}
$$

where $\left\{1\left(x_{\alpha}\right)\right\}$ converges to $e \neq 0$ and $g_{\alpha}$ converges to $g \in H(1)$. Then

$$
\begin{aligned}
\pi \psi\left(x_{\alpha}\right) & =\mu \phi\left(\prod_{i \in N} x_{i}^{p(i, \alpha)}\right)-\mu \phi\left(\prod_{i \in M} x_{i}^{p(i, \alpha)}\right) \\
& =\sum_{i \in N} p(i, \alpha) \mu \phi\left(x_{i}\right)-\sum_{i \in M} p(i, \alpha) \mu \phi\left(x_{i}\right) \\
& =\sum_{i \in N} p(i, \alpha) \mu\left(z_{i}\right)-\sum_{i \in M} p(i, \alpha) \mu\left(z_{i}\right) .
\end{aligned}
$$

If some subnet $\pi \psi\left(x_{\beta}\right)$ converges to $w \in R^{k}, w=\sum_{i \in N} p(i) \mu\left(z_{i}\right)-\sum_{i \in M} p(i) \mu\left(z_{i}\right)$, then, for each $i \in M \cup N$, the associated net $\{p(i, \beta)\}$ must converge to $p(i)$. Let $a=$ 
$\prod_{i \in M} x_{i}^{p(t)}, b=\prod_{i \in N} x_{i}^{p(i)}$. By continuity of multiplication in $S$, we have $0 a=b e g$, whence $e=0$ by the fifth reduction. This contradiction establishes that the net $\left\{\pi \psi\left(x_{\alpha}\right)\right\}$ converges to $\infty$.

Now, for $e \in E(S) \mid\{0\}$, let $Q(e)=\phi^{-1}(P(\phi(e))) \mid\{0\}$. If $t \in S$ such that $t a=b e g$, with $a, b \in Y \backslash\{0\}$ and $g \in H(1)$, then clearly $t \in Q(e)$. Conversely, given any $t \in Q(e)$, there exist $a, b \in Y \backslash\{0\}$ and $g \in H(1)$ such that $t a=b 1(t) g$; it is easy to see that $1(t)$ must be $e$. Hence $Q(e)=\{t \in S$ : there exist $a, b \in Y \backslash\{0\}, g \in H(1)$ such that $t a=b e g\}$. Let $C(e)=\pi \psi(Q(e))$.

For each $e \in E(S)\left\{\{0\}, C(e)\right.$ is a closed cone in $R^{k}$. Let $\left\{\pi \psi\left(x_{\alpha}\right)\right\}, x_{\alpha} \in Q(e)$, be a net in $C(e)$ converging to $z \in R^{k}$. Let $\left\{a_{\alpha}\right\},\left\{b_{\alpha}\right\}$ be nets in $Y \backslash\{0\}$ and let $\left\{g_{\alpha}\right\}$ be a net in $H(1)$ such that $x_{\alpha} a_{\alpha}=b_{\alpha} e g_{\alpha}$ for all $\alpha$. Since $\left\{\pi \psi\left(x_{\alpha}\right)\right\}$ does not converge to $\infty$ in $R^{k}$, the net $\left\{x_{\alpha}\right\}$ cannot cluster to 0 in $S$. Hence by choosing subnets as before, we may assume convergence of the nets $\left\{x_{\alpha}\right\}$ to $t \neq 0,\left\{g_{\alpha}\right\}$ to $g \in H(1),\left\{a_{\alpha}\right\}$ to $a \in Y \backslash\{0\}$, and $\left\{b_{\alpha}\right\}$ to $b \in Y \backslash\{0\}$. Thus $t a=b e g$, so that $t \in Q(e)$. Finally, $\left\{\pi \psi\left(x_{\alpha}\right)\right\}=\left\{\mu \phi\left(b_{\alpha}\right)\right.$ $\left.-\mu \phi\left(a_{\alpha}\right)\right\}$, which converges to $\mu \phi(b)-\mu \phi(a)$, which is $\pi \psi(t)$, so that $z=\pi \psi(t) \in C(e)$. Hence $C(e)$ is closed in $R^{k}$. It is easily deduced that $C(e)$ is an additive subsemigroup, closed under multiplication by nonnegative real numbers. To complete the claim, let $z=\pi \psi(t),-z=\pi \psi(s)$ belong to $C(e)$. We show $z=0$. Indeed, if $t a=b e g$ and $s c=d e h$, then $\phi(a c)=\phi(b d)$, whence $a c=b d$. Thus $t s b d=t s a c=b d e g h$, so by Lemma $3 t s=e g h \in H(e)$, and hence $t \in H(e)$. This established $\phi(a)=\phi(b)$, and therefore $z=0$.

In particular, $C(1)$ is a closed cone in $R^{k}$. By Theorem 1.6 of [6], there exists a nonsingular linear transformation of $R^{k}$ onto $R^{k}$ which maps $C(1)$ into the nonnegative cone of $R^{k}$. Hence we may assume $C(1)$ is already a subset of the nonnegative cone of $R^{k}$. Let $q$ be the vector in $R^{k}$, each of whose coordinates is 1 . Let $c$ be the minimum of the usual inner product $\langle v, q\rangle$ restricted to the compact set of elements in $C(1)$ having norm 1. Clearly $c>0$, and for any $v$ in $C(1)$ we have $c\|v\| \leqq\langle v, q\rangle$. Let $C=\left\{v \in R^{k}:(c / 2)\|v\| \leqq\langle v, q\rangle\right\}$. It is easily seen that $C$ is a closed cone in $R^{k}$.

There exists an open set $U$ in $S$, containing 1 , such that if $e \in E \cap U$, then $C(e) \subset C$. If not, there is a net in $S$ of idempotents $\left\{e_{\alpha}\right\}$ converging to 1 and a net $\left\{z_{\alpha}\right\}$ in $R^{k}$ such that $z_{\alpha} \in C\left(e_{\alpha}\right) \mid C$. Clearly, we may assume $\left\|z_{\alpha}\right\|=1$ for each $\alpha$. Let $x_{\alpha} \in Q\left(e_{\alpha}\right)$ such that $\pi \psi\left(x_{\alpha}\right)=z_{\alpha}$. Let $\left\{x_{\alpha}\right\}$ cluster to $t$. Note $t \neq 0$, since $\left\{e_{\alpha}\right\}$ does not cluster to 0 , and each $z_{\alpha}$ has norm 1. As in the proof that each $C(e)$ is closed, we obtain nets $\left\{a_{\alpha}\right\},\left\{b_{\alpha}\right\}$ in $Y \backslash\{0\},\left\{g_{\alpha}\right\}$ in $H(1)$ such that $x_{\alpha} a_{\alpha}=b_{\alpha} e_{\alpha} g_{\alpha}$, and thus elements $a, b \in Y \backslash\{0\}, g \in H(1)$ such that $t a=b g$. This shows $t \in Q(1)$ and hence $\pi \psi(t) \in C(1)$. However, by continuity of the usual inner product, this is impossible. Hence such an open set must exist, and it is no loss of generality to assume $0 \notin U$.

Let $F=E(S) \cap(S \backslash U)$. The semigroup $S / S F$ satisfies all the properties that $S$ satisfies, and the images of the original $x$ and $y$ remain distinct. Let $\hat{\psi}: S / S F \mid\{0\}$ $\rightarrow R^{k} \times E(S / S F) \times H(1)$ be defined in the same way that $\psi$ was defined on $S \backslash\{0\}$. For any $z \in S / S F \mid\{0\}$, the preimage $w$ of $z$ belongs to $S \backslash S F$, so that $1(w) \in U$ and 
hence $\pi \psi(w) \in C$. Since the natural map from $S$ to $S / S F$ leaves $S \backslash S F$ undisturbed, we thus have $\hat{\pi} \hat{\psi}(z) \in C$, where $\hat{\pi}$ is the projection of $\hat{\psi}(S / S F \mid\{0\})$ into $R^{k}$. Hence we may consider that $\hat{\psi}$ maps $S / S F \backslash\{0\}$ into $C \times E(S / S F) \times H(1)$.

The cone $C$ can be compactified by the addition of an ideal point $\{\omega\}$ which acts as a zero [14]. We denote this compactified cone by $C^{k}$. In the semigroup $C^{k} \times E(S / S F) \times H(1)$, let $I=\left(C^{k} \times\{0\} \times H(1)\right) \cup(\{\omega\} \times E(S / S F) \times H(1))$. Clearly $I$ is a closed ideal.

Let $\eta$ be the natural homomorphism of $C^{k} \times E(S / S F) \times H(1)$ onto the Rees quotient semigroup determined by $I$. Since $\hat{\psi}(S / S F \mid\{0\})$ and $I$ are disjoint, $\eta \hat{\psi}$ is a continuous isomorphism. We extend this map to ext $(\eta \hat{\psi})$ by sending the zero of $S / S F$ into the zero of $C^{k} \times E(S / S F) \times H(1)$ modulo $I$. It has been shown above that if $\left\{x_{\alpha}\right\}$ is a net in $S / S F \mid\{0\}$ converging to 0 , then either $\left\{1\left(x_{\alpha}\right)\right\}$ clusters to 0 or $\left\{\pi \psi\left(x_{\alpha}\right)\right\}$ converges to $\infty$. Hence ext $(\eta \hat{\psi})$ is continuous on $S / S F$. It is directly computable that ext $(\eta \hat{\psi})$ is an isomorphism. Now consider the following diagram:

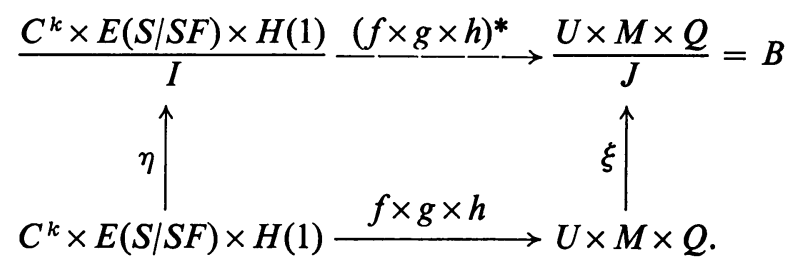

Here, $f \in \operatorname{Hom}\left(C^{k}, I\right), g \in \operatorname{Hom}(E, M)$, and $h \in \operatorname{Hom}(H(1), Q)$, and $\eta$ and $\xi$ are natural homomorphisms. It is clear from the definitions of $I$ and $J$ that for each triple $(f, g, h)$ there is an induced homomorphism $(f \times g \times h)^{*}$ as indicated above. It is easy to see that the family of homomorphisms $\left\{(f \times g \times h)^{*}\right\}$ separates the points of $C^{k} \times E(S / S F) \times H(1) / I$ and hence those of ext $(\eta \hat{\psi})(S / S F)$. This completes the proof.

3. Corollaries and conjectures. Let $G$ be the circle group, and let $D$ be the Rees quotient semigroup of $C \times M \times G$ modulo the ideal $K$ of all triples $(c, m, g)$ such that $c=0$ or $m=0$.

Corollary 1. Hom $(B, D)$ separates points.

Proof. Let $x$ and $y$ be distinct elements of $B$. Consider the diagram

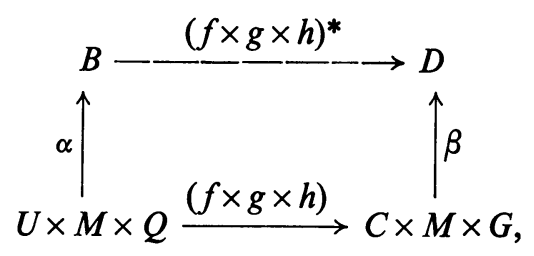

where $f \in \operatorname{Hom}(U, C), g \in \operatorname{Hom}(M, M), h \in \operatorname{Hom}(Q, G), \alpha$ and $\beta$ are natural maps, and $(f \times g \times h)^{*}$ is induced from $B$ into $D$. Let $x^{\prime}=(u, m, q), y^{\prime}=(v, n, r)$ such that $\alpha\left(x^{\prime}\right)=x$ and $\alpha\left(y^{\prime}\right)=y$. Since $x \neq y$, these triples differ in at least one 
coordinate and either $u \neq 0 \neq m$ or $v \neq 0 \neq n$. Since each of $\operatorname{Hom}(U, C), \operatorname{Hom}(M, M)$, and $\operatorname{Hom}(Q, C)$ separates points, it is now easy to select a triple $(f, g, h)$ such that $\beta \cdot(f \times g \times h)\left(x^{\prime}\right) \neq \beta \cdot(f \times g \times h)\left(y^{\prime}\right)$.

If $x \in L$, a semilattice, then $M(x)=\{y \in L: x y=x\}$. If $L$ is a compact topological semilattice, then each $M(x)$ is a compact subsemilattice of $L$.

CoRollary 2. Let $S$ be a finite-dimensional compact UDC such that, for each $x$ in the semilattice $E(S), M(x)$ is a connected set. Then $\operatorname{Hom}(S, B)$, and hence Hom $(S, D)$, separates points.

Proof. Lawson has showed in [20] that if $L$ is a finite-dimensional compact semilattice with $M(x)$ connected for each $x$, then $\operatorname{Hom}(L, M)$ separates points.

We conclude with the following conjectures, not all of which are new. In each conjecture, the hypothesis "finite-dimensional" can be deleted to form a (presumably) more difficult problem.

(1) If $L$ is a (finite-dimensional) compact semilattice, then $\operatorname{Hom}(L, M)$ separates points.

(2) If $S$ is a (finite-dimensional) compact Abelian divisible semigroup, then Hom $(S, D)$ separates points.

(3) If $S$ is a (finite-dimensional) compact Abelian semigroup, then $S$ is iseomorphically embeddable in a (finite-dimensional) compact Abelian divisible semigroup.

\section{REFERENCES}

1. L. W. Anderson, On the breadth and co-dimension of a topological lattice, Pacific J. Math. 9 (1959), 715-720.

2. - The existence of continuous lattice homomorphisms, J. London Math. Soc. 37 (1962), 60-62.

3. L. W. Anderson and R. P. Hunter, Homomorphisms and dimension, Math. Ann. 147 (1962), 248-268.

4. - , The $\mathscr{H}$-equivalence in compact semigroups, Bull. Soc. Math. Belg. 14 (1962), 274-296.

5. - The $\mathscr{H}$-equivalence in compact semigroups. II, J. Austral. Math. Soc. 3 (1963), 288-293.

6. D. R. Brown and M. Friedberg, Representation theorems for uniquely divisible semigroups, Duke Math. J. 35 (1968), 341-352.

7. D. R. Brown and J. G. LaTorre, A characterization of uniquely divisible commutative semigroups, Pacific J. Math. 18 (1966), 57-60.

8. C. E. Clark, Locally algebraically independent collections of sub-semigroups of a semigroup, Duke Math. J. 35 (1968), 843-851.

9. A. H. Clifford and G. B. Preston, The algebraic theory of semigroups, Vol. I, Math. Surveys No. 7, Amer. Math. Soc., Providence, R. I., 1962.

10. E. Hewitt and K. A. Ross, Abstract harmonic analysis, Academic Press, New York, 1963.

11. J. A. Hildebrant, On compact unithetic semigroups, Pacific J. Math. 21 (1967), 265-273.

12. - On uniquely divisible semigroups on the two-cell, Pacific J. Math. 23 (1967), 91-95. 
13. J. A. Hildebrant, On compact divisible abelian semigroups, Proc. Amer. Math. Soc. 19 (1968), 405-410.

14. K. H. Hoffman and P. S. Mostert, Elements of compact semigroups, Merrill, Columbus, Ohio, 1966.

15. Anne (Lester) Hudson, Some semigroups on the two-cell, Proc. Amer. Math. Soc. 10 (1959), 648-655.

16. R. P. Hunter and N. J. Rothman, Characters and cross-sections for certain semigroups, Duke Math. J. 29 (1962), 347-366.

17. K. Keimel, Lokal kompakte kegelhalbgruppen und deren Einbettung in topologische Vektorraume (to appear).

18. R. J. Koch, Monothetic semigroups, Proc. Amer. Math. Soc. 8 (1957), 397-401.

19. - Ordered semigroups in partially ordered semigroups, Pacific J. Math. 10 (1960), 1333-1336.

20. J. D. Lawson, Vietoris mappings and embeddings of topological semi-lattices, Dissertation, University of Tennessee, 1967.

21. P. S. Mostert and A. L. Shields, On the structure of semigroups on a compact manifold with boundary, Ann. of Math. 64 (1967), 117-143.

22. —_, One parameter semigroups in a semigroup, Trans. Amer. Math. Soc. 96 (1960), 510-517.

23. Stefan Schwarz, The theory of characters of commutative Hausdorff bicompact semigroups, Czechoslovak Math. J. 6 (1956), 330-361.

24. A. D. Wallace, The structure of topological semigroups, Bull. Amer. Math. Soc. 61 (1955), 95-112.

UNIVERSITY OF HOUSTON, Houston, TeXas 